\title{
ABSOLUTE CONJUGATE FOURIER EFFECTIVE METHODS AND FUNCTIONS
}

\author{
A. KUMAR AND A. PEYERIMHOFF
}

(Communicated by J. Marshall Ash)

\begin{abstract}
We consider matrix transformation of the conjugate series of a Fourier series. Necessary and sufficient conditions on the function, generating the Fourier series, as well as on the method of the transformation have been obtained for absolute summability of the transformed series.
\end{abstract}

\section{INTRODUCTION AND MAIN RESULT}

Let $\hat{A}=\left(\hat{a}_{n, k}\right)$ be the matrix of a series-to-series transformation, where $\hat{a}_{n, k}$ are real or complex numbers. A series $\sum_{n \geq 0} a_{n}$ is said to be summable $\hat{A}$ if $\alpha_{n}=\sum_{k \geq 0} \hat{a}_{n, k} a_{k}$ exists for all $n$ and $\sum_{n \geq 0} \alpha_{n}$ is convergent. If $\alpha_{n}$ exists for all $n$ and $\sum_{n \geq 0}\left|\alpha_{n}\right|<\infty$, then the series $\sum_{n \geq 0} a_{n}$ is said to be absolutely summable $\hat{A}$ or $|\hat{A}|$ summable. The matrix $\hat{A}$ is absolutely conservative, i.e., it transforms an absolutely convergent series to an absolutely convergent series if $\sum_{n \geq 0}\left|\hat{a}_{n, k}\right|=0(1)$ for all $k$. Let $p=\left\{p_{n}\right\}$ be the sequence of constants, real or complex, such that $P_{n}=p_{0}+p_{1}+\cdots+p_{n} \neq 0$. The Nörlund matrix, denoted by $(\widehat{N}, p)$, is the matrix $\hat{A}$ with $\hat{a}_{n, k}=P(n, k)=P_{n-k} / P_{n}-P_{n-k-1} / P_{n-1}$ for $0<k \leq n, P(0,0)=0$, and $P(n, k)=0$ for $k>n$. The matrix $(\hat{N}, p)$ reduces to the $(C, \alpha)$ matrix if

$$
p_{n}=A_{n}^{\alpha-1}, \quad \text { where }(1-z)^{-\alpha-1}=\sum_{n \geq 0} A_{n}^{\alpha} z^{n} \quad(|z|<1, \alpha>-1) .
$$

In what follows we write $\Delta p_{n}=p_{n}-p_{n+1} ; S$ and $T$ denote the class of sequences $p$ for which $\sum_{n \geq k} 1 /\left(n\left|P_{n}\right|\right)=O\left(1 / P_{k}\right), k=1,2,3, \ldots$, and $\sum_{k=1}^{n} k\left|\Delta p_{k}\right|=O\left(P_{n}\right)$, respectively; $P_{n}^{*}=\sum_{k=0}^{n}\left|p_{k}\right| ; K$ denotes a positive constant which is not necessarily the same at each occurrence.

Let $f$ be an $L$ integrable, periodic function with period $2 \pi$. BV denotes the set of functions of bounded variation in the interval $(0,2 \pi)$. We say $f \in$ IC if

$$
\int_{0}^{\pi}|\psi(t)| t^{-1} d t<\infty, \quad \psi(t)=f(x+t)-f(x-t) .
$$

Received by the editors October 7, 1991 and, in revised form, November 9, 1992. 1991 Mathematics Subject Classification. Primary 40C05, 42A50. 
By $\widehat{A} \in(\mathrm{BV} \rightarrow|F|)$ we mean that the Fourier series $\sum_{n \geq 0} A_{n}(x)$ of $f(x)$, $f \in \mathrm{BV}$, is $|\widehat{A}|$ summable. Similarly, $\widehat{A} \in(\mathrm{BV} \rightarrow|\widetilde{F}|)$ means that the conjugate Fourier series $\sum_{n \geq 0} B_{n}(x)$ of $f(x), f \in \mathrm{BV}$, is $|\widehat{A}|$ summable.

Concerning $(\tilde{N}, p) \in(\mathrm{BV} \rightarrow|\widetilde{F}|)$, Pati [7] proved

Theorem A. Let the positive sequence $p$ be such that $\left\{(n+1) p_{n} / P_{n}\right\}$ and $\left\{\sum_{k=0}^{n} P_{k} /\left(k P_{n}\right)\right\}$ are of bounded variation. Then $(\widehat{N}, p) \in(B V \rightarrow|\widetilde{F}|)$, if $f \in I C$.

For the Fourier series the following is known ([1, Theorem G]; see also [3, 4, 6]).

Theorem B. Let $p \in S \cap T$. Then $(\widehat{N}, p) \in(B V \rightarrow|F|)$.

From Theorems A and B we see that in the former case an additional condition on $f$, viz. $f \in \mathrm{IC}$, is assumed. The requirement of this additional condition for a particular case was shown by Kumar [5] in the following form.

Theorem C. Let $p$ be a nonnegative nonincreasing sequence $\in S$. Then $(\widehat{N}, p)$ $\in(B V \rightarrow|\widetilde{F}|) \Leftrightarrow f \in I C$.

A necessary condition on $p$ such that $(\widehat{N}, p) \in(\mathrm{BV} \rightarrow|F|)$ has been investigated in [4, Theorem 8]. In the present paper we investigate what are necessary conditions on the sequence $p$ and the function $f$ for $(\widehat{N}, p) \in(\mathrm{BV} \rightarrow|\widetilde{F}|)$. We first consider a general series-to-series matrix transformation and obtain

Theorem 1. If $\hat{A} \in(B V \rightarrow|\widetilde{F}|)$, then

$$
\sum_{\substack{n \\ r(n) \geq m}}\left|\sum_{\substack{k \\ r(n)-m \leq k \leq r(n)+m}} \frac{\hat{a}_{n}, k}{k}\left(1-\frac{|r(n)-k|}{m+1}\right)\right|=O(1), \quad m \geq 1,
$$

where $r(n)$ is any sequence contained in $\mathbb{N}_{0}$.

\section{LEMMAS}

We make use of the following results in this paper.

Lemma 1. Let $\psi(t)$ be a function of bounded variation in $[0, \pi]$, and let for $n>1$

$$
E_{n}=\{t:|\psi(t)| \text { is continuous at } t \in[\pi / n, \pi /(n-1))\} .
$$

Then there exist $\theta_{n}$ and $\theta_{n}^{\prime} \in E_{n}$ such that for all $x \in E_{n}$

$$
\left|\psi\left(\theta_{n}\right)\right|-\frac{1}{n} \leq|\psi(x)| \leq\left|\psi\left(\theta_{n}^{\prime}\right)\right|+\frac{1}{n},
$$

and

$$
\sum_{n>2}\left|\psi\left(\theta_{n}\right)\right| n^{-1}<\infty \Leftrightarrow \sum_{n>2}\left|\psi\left(\theta_{n}^{\prime}\right)\right| n^{-1}<\infty \Leftrightarrow f \in I C .
$$


Lemma 1 is contained in [5, Lemma 4].

Lemma 2. Let $p \in T$. Then

(i) $\sum_{n \geq k}|P(n, k)| \leq K, k>0$;

(ii) $n p_{n}=O\left(P_{n}\right)$;

(iii) $P_{2 n} \sim P_{n} \sim P_{n}^{*}$;

(iv) $\sum_{n \geq \mu}|P(n, k)| \leq K k(\mu-k+1)^{-1}, \mu \geq k \geq 1$;

(v) $\sum_{k=1}^{n}|P(n, k)|=O(1)$.

Proof. That the results (i) and (ii) and the fact that $P_{n}^{*}=O\left(P_{n}\right)$ follow from $p \in T$ are contained in [1, Lemmas 4 and 1(b)]. In view of the result [2, Lemma 1], (iii) follows from (ii) and the fact that $P_{n}^{*}=O\left(P_{n}\right)$. In order to prove (iv), we write

$$
\begin{aligned}
\sum_{n \geq \mu}|P(n, k)| \leq & \sum_{n \geq \mu}\left|\frac{\left(P_{n}-P_{n-k}\right)-\left(P_{n-1}-P_{n-k-1}\right)}{P_{n}}\right| \\
& +\sum_{n \geq \mu}\left|\frac{p_{n}\left(P_{n-1}-P_{n-k-1}\right)}{P_{n} P_{n-1}}\right| \\
\leq & \sum_{n \geq \mu} \sum_{r=n-k}^{n-1}\left|\frac{\Delta p_{r}}{P_{n}}\right|+K \sum_{n \geq \mu} \frac{1}{n\left|P_{n}\right|} \sum_{r=1}^{k}\left|p_{n-r}\right| .
\end{aligned}
$$

We consider a typical term, i.e., the term corresponding to $r=n-j, 1 \leq j \leq k$, of the first sum. In virtue of Lemma 2(iii) and (ii), we have $\left|\Delta\left[1 /\left((n+1) P_{n}^{*}\right)\right]\right| \leq$ $K /\left|(n+1)^{2} P_{n}\right|$ and $P_{n+j} \sim P_{n+j}^{*} \sim P_{n}^{*}$. For $N \rightarrow \infty$, we obtain by Abel's transformation

$$
\begin{aligned}
\sum_{n=\mu}^{N}\left|\frac{\Delta p_{n-j}}{P_{n}}\right| \leq & K \sum_{n=\mu-j}^{N-j} \frac{\left|\Delta p_{n}\right|}{P_{n}^{*}} \\
\leq & K \sum_{n=\mu-j}^{N-j} \frac{1}{(n+1)^{2}\left|P_{n}\right|} \sum_{k=1}^{n}(k+1)\left|\Delta p_{k}\right| \\
& +\frac{1}{(N-j+2) P_{N-j+1}^{*}} \sum_{k=1}^{N-j}(k+1)\left|\Delta p_{k}\right| \\
& +\frac{1}{(\mu-j+1) P_{\mu-j}^{*}} \sum_{k=1}^{\mu-j-1}(k+1)\left|\Delta p_{k}\right| \\
\leq & \frac{K}{(\mu-j+1)},
\end{aligned}
$$

since $p \in T$. In view of this and Lemma 2(ii), we get

$$
\sum_{n \geq \mu}|P(n, k)| \leq K k /(\mu-k+1)+K k \sum_{n \geq \mu} 1 /(n(n-k+1)) \leq K k /(\mu-k+1),
$$

since $\sum_{n \geq \mu} 1 /(n(n-k+1)) \leq 2 \sum_{n \geq \mu} 1 /((n-k+2)(n-k+1))=2 /(\mu-k+1)$.

The last part follows from Lemma 2(ii) and (iii) and the identity $P(n, k)=$ $p_{n-k} / P_{n}-p_{n} P_{n-k-1} /(P n P n-1)$. This completes the proof of the lemma. 


\section{Proof of Theorem 1}

Writing

$$
B_{k}(x)=\frac{2}{\pi} \int_{0}^{\pi} \psi(t) \sin k t d t=-\frac{2}{k \pi} \int_{0}^{\pi}(1-\cos k t) d \psi(t),
$$

we see that $\widehat{A} \in(\mathrm{BV} \rightarrow|\widetilde{F}|)$ if and only if

$$
\sum_{n \geq 1}\left|\sum_{k \geq 1} \frac{\hat{a}_{n, k}}{2 k} \int_{0}^{\pi} d \psi(t) \sin ^{2} \frac{k t}{2}\right|<\infty .
$$

Define $\phi_{n, m}(f)=\sum_{k=1}^{m} \hat{a}_{n, k} B_{k}(0)$,

$$
f_{t}(x)=\sum_{k=1}^{\infty} \frac{1-\cos k t}{2 k} \sin k x=\sum_{k=1}^{\infty} \frac{\sin ^{2}(k t / 2)}{k} \sin k x .
$$

Then (cf. $[4$, p. 102])

$$
\left|\phi_{n, m}(f)\right| \leq C_{n} \operatorname{Var} f
$$

and hence

$$
\left|\sum_{k=1}^{m} \frac{\hat{a}_{n, k}}{k} \sin ^{2} \frac{k t}{2}\right| \leq c C_{n}
$$

where $c=\sup _{t} \operatorname{Var} f_{t}<\infty$. Since $\hat{A} \in(\mathrm{BV} \rightarrow|\widetilde{F}|)$, we have

$$
\sum_{n \geq 1}\left|\lim _{m} \phi_{n, m}(f)\right|<\infty
$$

for every $2 \pi$ periodic $f \in \mathrm{BV}$. Hence, by the Banach-Steinhaus theorem, $\sum_{n} \varepsilon_{n} \lim _{m} \phi_{n, m}(f)$, where $\varepsilon_{n} \in\{1,-1\}$, is a bounded linear functional in $\mathrm{BV}$, so

$$
\sum_{n \geq 1}\left|\lim _{m} \phi_{n, m}(f)\right| \leq D \operatorname{Var} f \text { for some } D>0 .
$$

Replacing $f$ by $f_{t}$ in this inequality yields

$$
\sup _{t} \sum_{n \geq 1}\left|\sum_{k \geq 1} \frac{\hat{a}_{n, k}}{k} \sin ^{2} \frac{k t}{2}\right|<\infty .
$$

Let $K_{m}(x)=\frac{1}{2}+\sum_{i=1}^{m}\left(1-\frac{i}{m+1}\right) \cos i x$. We have

$$
\cos (r(n) x) K_{m}(x)=\sum_{i=r(n)-m}^{r(n)+m}\left(1-\frac{|r(n)-i|}{m+1}\right) \frac{\cos i x}{2} .
$$

For every sequence $\left\{\varepsilon_{n}\right\}$ and for all large values of $N$ we write

$$
\begin{aligned}
I & =\int_{0}^{\pi} K_{m}(t)\left(\sum_{n=1}^{N} \varepsilon_{n} \cos (r(n) t) \sum_{k \geq 1} \frac{\hat{a}_{n, k}}{k}(1-\cos k t)\right) d t \\
& =\frac{\pi}{8} \sum_{n=1}^{N} \varepsilon_{n} \sum_{k=r(n)-m}^{r(n)+m} \frac{\hat{a}_{n, k}}{k}\left(1-\frac{|k-r(n)|}{m+1}\right),
\end{aligned}
$$


since $\int_{0}^{\pi} \cos$ it $\sin ^{2}(k t / 2) d t=\frac{\pi}{4}$ for $i=k$, and zero otherwise. By the fact that the integral of $K_{m}(t)$ over $(-\pi, \pi)=\pi$, we see that (2) implies that $I=O(1)$. The theorem follows as $I=O(1)$ for every choice of $\left\{\varepsilon_{n}\right\}$.

\section{THEOREM 2 AND ITS PROOF}

Using the above result we prove the following for the Nörlund matrix. For the sake of convenience we say $p \in S^{\prime}$ if

$$
\sum_{n \geq 2 m} \frac{1}{m\left|P_{n}\right|}\left|\sum_{k=0}^{m} \frac{P_{k}}{n-k}\right|=O(1), \quad m=1,2, \ldots
$$

Theorem 2. Let $p \in T$. Then

(i) $(\widehat{N}, p) \in(B V \rightarrow|\widetilde{F}|) \Rightarrow p \in S^{\prime}$;

(ii) $(\widehat{N}, p) \in(B V \rightarrow|\widetilde{F}|) \Rightarrow f \in I C$ if $p \in S$;

(iii) $f \in I C$ and $p \in S \Rightarrow(\widehat{N}, p) \in(B V \rightarrow|\widetilde{F}|)$.

Remark. If $p$ is real, $p \in S^{\prime}$ is equivalent to $p \in S$ for $p \in T$, because (cf. [4, p. 112]) the sequence $\left\{P_{n}\right\}$, for sufficiently large $n$, has a fixed sign. In view of Lemma 2(iii), (3) implies

$$
\sum_{n \geq 2 m} \frac{1}{m\left|P_{n}\right|} \sum_{k=0}^{m} \frac{P_{k}^{*}}{n-k}=O(1)
$$

and this gives that

$$
\sum_{n \geq 2 m} \frac{P_{[m / 2]}^{*}}{m n\left|P_{n}\right|} \sum_{k=[m / 2]}^{m} 1=O(1) .
$$

This is equivalent to $p \in S$ by Lemma 2(iii). It is direct to see that, even if $p$ is complex, $p \in S$ implies $p \in S^{\prime}$. Thus, if $p$ is real and $\in T$, then

$$
(\widehat{N}, p) \in(\mathrm{BV} \rightarrow|\widetilde{F}|) \Leftrightarrow f \in \mathrm{IC} \text { and } p \in S .
$$

Proof of Theorem 2. Letting $r(n)=n$ and $m=\tau=[\pi / t]$ in Theorem 1, we find that a necessary condition for $(\widehat{N}, p) \in(\mathrm{BV} \rightarrow|\widetilde{F}|)$ is

$$
\sum=\sum_{n \geq 2 \tau}\left|\sum_{k=n-\tau}^{n} \frac{P(n, k)}{k}\left(1-\frac{n-k}{\tau+1}\right)\right|=O(1) .
$$

Writing $P(n, k)=p_{n-k} / P_{n}-P_{n-k-1} p_{n} /\left(P_{n} P_{n-1}\right)$ and using that $n p_{n}=O\left(P_{n}\right)$, we get

$$
\sum \geq \sum_{n \geq 2 \tau} \frac{1}{(\tau+1)\left|P_{n}\right|}\left|\sum_{k=0}^{\tau} \frac{(\tau-k+1) p_{k}}{n-k}\right|-\sum_{n \geq 2 \tau} \frac{K}{n\left|P_{n}\right|} \sum_{k=0}^{\tau} \frac{(\tau-k+1)\left|P_{k-1}\right|}{(n-k)(\tau+1)} .
$$

By Lemma 2(iii), the second sum $\leq K \sum_{n \geq 2 \tau} \tau /((n-\tau) n)=O(1)$. Since

$$
\sum_{k=0}^{\tau} \frac{\tau-k+1}{n-k} p_{k}=\sum_{k=0}^{\tau}\left(\frac{\tau-k+1}{n-k}-\frac{\tau-k}{n-k-1}\right) P_{k}
$$


we get

$$
\begin{aligned}
\sum \geq & \sum_{n \geq 2 \tau} \frac{1}{(\tau+1)\left|P_{n}\right|}\left|\sum_{k=0}^{\tau} \frac{P_{k}}{n-k}\right| \\
& -\sum_{n \geq 2 \tau} \frac{1}{(\tau+1)\left|P_{n}\right|} \sum_{k=0}^{\tau} \frac{(\tau-k)\left|P_{k}\right|}{(n-k)(n-k-1)}-O(1) .
\end{aligned}
$$

Again the second sum is bounded by virtue that $P_{n}^{*}=O\left(P_{n}\right)$. Since $\sum=O(1)$, we get that $p \in S^{\prime}$.

In order to prove the necessity of $f \in I C$, we write

$$
B_{k}(x)=-\frac{4}{k \pi} \int_{0}^{\theta_{n}} \sin ^{2} \frac{k t}{2} d \psi(t)+\frac{2 \psi\left(\theta_{n}\right)}{k \pi}+\frac{2}{k \pi} \int_{\theta_{n}}^{\pi} \cos k t d \psi(t),
$$

where the $\theta_{n}$ 's are numbers considered in Lemma 1.

We proceed to estimate

$$
\begin{aligned}
& \sum_{1}=\sum_{n>1}\left|\int_{0}^{\theta_{n}}\left\{\sum_{k=1}^{n} \frac{P(n, k)}{k} \sin ^{2} \frac{k t}{2}\right\} d \psi(t)\right|, \\
& \sum_{2}=\sum_{n>1}\left|\psi\left(\theta_{n}\right)\right|\left|\sum_{k=1}^{n} \frac{P(n, k)}{k}\right|, \\
& \sum_{3}=\sum_{n>1}\left|\int_{\theta_{n}}^{\pi}\left\{\sum_{k=1}^{n} \frac{P(n, k)}{k} \cos k t\right\} d \psi(t)\right| .
\end{aligned}
$$

Since $\psi(t) \in \mathrm{BV}, t<\pi /(n-1)$, and $|\sin \theta| \leq K \theta$, we get by Lemma 2(i)

$$
\begin{aligned}
\sum_{1} & \leq \int_{0}^{\pi}|d \psi(t)| \sum_{n=2}^{\tau+1}\left|\sum_{k=1}^{n} \frac{P(n, k)}{k} \sin ^{2} \frac{k t}{2}\right| \\
& \leq K \int_{0}^{\pi}|d \psi(t)| t^{2} \sum_{k=1}^{\tau+1} k \sum_{n=k}^{\tau+1}|P(n, k)| \leq K .
\end{aligned}
$$

Suppose $N$ is any arbitrary large number. In view of Lemma 2(i), we find that

$$
\begin{aligned}
& \sum_{2} \geq\left|\sum_{n=2}^{N}\right| \psi\left(\theta_{n}\right)\left|\sum_{k=2}^{n} \frac{P(n, k)}{k}\right|-\sum_{n=2}^{N}\left|\psi\left(\theta_{n}\right)\right||P(n, 1)| \\
&=\left|\sum_{k=2}^{N} \frac{1}{k} \sum_{n=k}^{N}\right| \psi\left(\theta_{n}\right)|P(n, k)|-O(1) \\
&=\left|\sum_{k=2}^{N} \frac{1}{k} \sum_{n=k}^{N} \Delta\right| \psi\left(\theta_{n}\right)\left|\frac{P_{n-k}}{P_{n}}+\sum_{k=2}^{N} \frac{\left|\psi\left(\theta_{N+1}\right)\right| P_{N-k}}{k P_{N}}\right|-O(1) \\
&=\left|\sum_{k=2}^{N} \frac{\left|\psi\left(\theta_{k}\right)\right|-\left|\psi\left(\theta_{N+1}\right)\right|}{k}-\sum_{k=2}^{N} \frac{1}{k} \sum_{n=k}^{N} \Delta\right| \psi\left(\theta_{n}\right) \mid \frac{\left(P_{n}-P_{n-k}\right)}{P_{n}} \\
&+\sum_{k=2}^{N} \frac{\left|\psi\left(\theta_{N+1}\right)\right| P_{N-k}}{k P_{N}} \mid-O(1)
\end{aligned}
$$




$$
\begin{aligned}
& \geq \sum_{k=2}^{N} \frac{\left|\psi\left(\theta_{k}\right)\right|}{k}-\sum_{n=2}^{N}\left|\Delta \psi\left(\theta_{n}\right)\right| \sum_{k=2}^{n}\left|\frac{P_{n}-P_{n-k}}{k P_{n}}\right| \\
& -\sum_{k=2}^{N}\left|\psi\left(\theta_{N+1}\right)\right|\left|\frac{P_{N}-P_{N-K}}{k P_{N}}\right|-O(1) .
\end{aligned}
$$

From Lemma 2(ii) and (iii) we see that

$$
\sum_{k=1}^{[n / 2]}\left|\frac{P_{n}-P_{n-k}}{k P_{n}}\right| \leq \sum_{k=1}^{[n / 2]} \frac{1}{k} \sum_{r=0}^{k-1}\left|\frac{p_{n-r}}{P_{n}}\right| \leq K \sum_{k=1}^{[n / 2]} \frac{1}{n-k} \leq K
$$

and

$$
\sum_{k=[n / 2]}^{n} \frac{\left|P_{n}-P_{n-k}\right|}{k\left|P_{n}\right|} \leq K \sum_{k=[n / 2]}^{n} \frac{1}{k} \leq K .
$$

Consequently, $\sum_{2} \geq \sum_{k=2}^{N} \frac{\left|\psi\left(\theta_{k}\right)\right|}{k}-O(1)$, since $\psi(t) \in \mathrm{BV}$. Hence from Lemma 1 we get

$$
\sum_{2}<\infty \Rightarrow f \in \mathrm{IC}
$$

Let $N$ be a number tending to $\infty$, and assume $f \in \mathrm{IC}$. We have

$$
\begin{aligned}
\sum_{2} & \leq \sum_{n=2}^{N}\left|\psi\left(\theta_{n}\right)\right| \sum_{k=1}^{[n / 2]} \frac{|P(n, k)|}{k}+\sum_{n=2}^{N} \frac{\left|\psi\left(\theta_{n}\right)\right|}{[n / 2]} \sum_{k=[n / 2]}^{n}|P(n, k)| \\
& \leq \sum_{k=1}^{[N / 2]} \frac{1}{k} \sum_{n=2 k}^{N}\left|\psi\left(\theta_{n}\right)\right||P(n, k)|+O(1)
\end{aligned}
$$

by Lemmas $2(\mathrm{v})$ and 1 . Writing the inner sum of the first term as

$$
\begin{aligned}
\sum_{n=2 k}^{N}\left|\psi\left(\theta_{n}\right) P(n, k)\right|= & -\sum_{n=2 k}^{N} \Delta\left|\psi\left(\theta_{n}\right)\right| \sum_{r=n+1}^{N+1}|P(r, k)| \\
& +\left|\psi\left(\theta_{2 k}\right)\right| \sum_{r=2 k}^{N+1}|P(r, k)|-\left|\psi\left(\theta_{N+1}\right) P(N+1, k)\right|
\end{aligned}
$$

and using Lemma 2(i), (iv), and (v), we obtain

$$
\begin{aligned}
\sum_{2} & \leq K \sum_{k=1}^{[N / 2]} \frac{1}{k} \sum_{n=2 k}^{N}\left|\Delta \psi\left(\theta_{n}\right)\right| \frac{k}{n-k}+K \sum_{k=1}^{N} \frac{\left|\psi\left(\theta_{2 k}\right)\right|}{k}+O(1) \\
& \leq K \sum_{n=1}^{N}\left|\Delta \psi\left(\theta_{n}\right)\right| \sum_{k=1}^{[n / 2]} \frac{1}{n-k}+O(1)=O(1) .
\end{aligned}
$$

To prove that $\sum_{3}<\infty$, it is sufficient to show that for $0<t \leq \pi$

$$
\sum_{n \geq \tau}\left|\sum_{k=1}^{n} P(n, k) k^{-1} \cos k t\right|=O(1) .
$$


We estimate the sum in (10) in parts. The following sum with $\tau=1$ is bounded by Lemma 2(i); for $\tau>1$ we get by Lemma 2(i) and (iv)

$$
\begin{aligned}
\sum_{n \geq \tau}\left|\sum_{k=1}^{\tau} \frac{P(n, k) \cos k t}{k}\right| & \leq\left(\sum_{k=1}^{[\tau / 2]}+\sum_{k=[\tau / 2]}^{\tau}\right) \frac{1}{k} \sum_{n \geq \tau}|P(n, k)| \\
& \leq \sum_{k=1}^{[\tau / 2]} \frac{1}{\tau-k}+K=O(1) .
\end{aligned}
$$

Also,

$$
\sum_{n=\tau}^{2 \tau}\left|\sum_{k=\tau+1}^{n} P(n, k) \frac{\cos k t}{k}\right| \leq \sum_{k=\tau}^{2 \tau} \frac{1}{k} \sum_{n=k}^{2 \tau}|P(n, k)|=O(1) .
$$

By Abel's transformation and Lemma 2(i), we have

$$
\begin{aligned}
& S=\sum_{n>2 \tau}\left|\sum_{k=\tau+1}^{n-\tau} P(n, k) \frac{\cos k t}{k}\right| \\
& \leq \frac{K}{t}\left\{\sum_{n>2 \tau} \sum_{k=\tau}^{n-\tau-1} \frac{1}{k}\left|\frac{p_{n-k}}{P_{n}}-\frac{p_{n-k-1}}{P_{n-1}}\right|+\sum_{n>2 \tau} \sum_{k=\tau}^{n-\tau} \frac{|P(n, k)|}{k^{2}}\right. \\
& \left.+\sum_{n>2 \tau} \frac{|P(n, \tau+1)|}{\tau}+\sum_{n>2 \tau} \frac{|P(n, n-\tau)|}{n-\tau}\right\} \\
& \leq \frac{K}{t}\left\{\sum_{n>2 \tau} \sum_{k=\tau}^{n-\tau}\left|\frac{p_{n-k}-p_{n-k-1}}{k P_{n}}\right|+\sum_{n>2 \tau} \sum_{k=\tau}^{n}\left|\frac{p_{n} p_{n-k-1}}{k P_{n} P_{n-1}}\right|\right. \\
& +\sum_{k \geq \tau} \frac{1}{k^{2}} \sum_{n \geq k}|P(n, k)|+\sum_{n>2 \tau}\left|\frac{p_{\tau}}{(n-\tau) P_{n}}\right| \\
& \left.+\sum_{n>2 \tau}\left|\frac{P_{\tau-1} p_{n}}{P_{n} P_{n-1}(n-\tau)}\right|\right\}+O(1) \\
& \leq \frac{K}{t}\left\{\sum_{n>2 \tau} \sum_{k=\tau}^{[n / 2]}\left|\frac{p_{n-k}-p_{n-k-1}}{k P_{n}}\right|\right. \\
& +\sum_{n>2 \tau} \frac{1}{n\left|P_{n}\right|} \sum_{k=[n / 2]}^{n-\tau}\left|p_{n-k}-p_{n-k-1}\right| \\
& \left.+\sum_{n>2 \tau} \sum_{k=\tau}^{[n / 2]}\left|\frac{p_{n} p_{n-k-1}}{k P_{n} P_{n-1}}\right|+\sum_{n>2 \tau} \sum_{k=[n / 2]}^{n}\left|\frac{p_{n} p_{n-k-1}}{k P_{n} P_{n-1}}\right|\right\} \\
& +K\left|P_{\tau}\right| \sum_{n>\tau} \frac{1}{n\left|P_{n}\right|}+O(1)
\end{aligned}
$$

by Lemma 2(ii) and (iii) and the fact that $\sum_{k \geq m>0} k^{-2} \leq 2 m^{-1}$. Applying change of the order of summation in the first term and the facts that $n p_{n}=$ 
$O\left(P_{n}\right)$ and $p \in S$, we obtain

$$
\begin{aligned}
S \leq \frac{K}{t}\left\{\sum_{k \geq \tau} \frac{1}{k} \sum_{n \geq 2 k}\left|\frac{p_{n-k}-p_{n-k-1}}{P_{n}}\right|+\sum_{n>2 \tau} \frac{1}{n\left|P_{n}\right|} \sum_{k=\tau}^{[n / 2]+1}\left|\Delta p_{k-1}\right|\right. \\
\left.+\sum_{k \geq \tau} \frac{1}{k} \sum_{n \geq 2 k} \frac{1}{n(n-k)}+\sum_{n \geq 2 \tau} \frac{1}{n^{2}} \sum_{k=[n / 2]}^{n}\left|\frac{p_{n-k-1}}{P_{n-1}}\right|\right\}+O(1) .
\end{aligned}
$$

Since $P_{n}^{*}=O\left(P_{n}\right)$, we have

$$
\begin{aligned}
S & \leq \frac{K}{t}\left\{\sum_{k \geq \tau} \frac{1}{k} \sum_{n \geq k}\left|\frac{\Delta p_{n-1}}{P_{n}}\right|+\sum_{k \geq \tau}\left|\Delta p_{k-1}\right| \sum_{n \geq k} \frac{1}{n\left|P_{n}\right|}+\sum_{k \geq \tau} \frac{1}{k^{2}}\right\}+O(1) \\
& =O(1)
\end{aligned}
$$

by the following inequality obtained in the proof of Lemma 2 and the fact that $p \in S$

$$
\sum_{n \geq \mu} \frac{\left|\Delta p_{n-1}\right|}{\left|P_{n}\right|} \leq \frac{K}{\mu}, \quad \mu \geq 1
$$

Lastly,

$$
\begin{aligned}
& \sum_{n>2 \tau}\left|\sum_{k=n-\tau+1}^{n} P(n, k) \frac{\cos k t}{k}\right| \leq K \sum_{n \geq 2 \tau} \frac{1}{n} \sum_{k=n-\tau}^{n}|P(n, k)| \\
& \quad \leq K \sum_{n \geq 2 \tau} \frac{1}{n} \sum_{k=n-\tau}^{n}\left|\frac{p_{n-k}}{P_{n}}\right|+K \sum_{n \geq 2 \tau} \frac{1}{n} \sum_{k=n-\tau}^{n}\left|\frac{p_{n} P_{n-k-1}}{P_{n} P_{n-1}}\right| \\
& \quad \leq K P_{\tau^{*}} \sum_{n \geq 2 \tau} \frac{1}{\left(n\left|P_{n}\right|\right)}+K \sum_{n \geq 2 \tau} \frac{(\tau+1)}{n^{2}}=O(1),
\end{aligned}
$$

by virtue of $p \in S$. Combining (11)-(14), we get (10). Hence

$$
\sum_{3}<\infty \text {. }
$$

From (5) and (6) we can see that the expression in (1) lies between $\left|\sum_{2}\right|-$ $\left|\sum_{1}\right|-\left|\sum_{3}\right|$ and $\left|\sum_{1}\right|+\left|\sum_{2}\right|+\left|\sum_{3}\right|$. Further, (7) and (15) give that $\sum_{1}+\sum_{3}<\infty$ if $p \in T \cap S$, while (8) and (9) imply that $\sum_{2}<\infty$ if and only if $f \in I C$ whenever $p \in T \cap S$. Hence we get (ii) and (iii) of the theorem.

This completes the proof of the theorem.

Combining [4, Theorem 8 and Remarks, p. 112] and (4), we obtain

Theorem 3. Let $p$ be real and $\in T$. Then

$$
(\widehat{N}, p) \in(B V \rightarrow|\widetilde{F}|) \Leftrightarrow(\widehat{N}, p) \in(B V \rightarrow|F|) \text { and } f \in I C .
$$

\section{ACKNOWLEDGMENT}

The authors are thankful to the referee Professor D. Borwein for his valuable suggestions in the proof of Theorem 1 and some other helpful suggestions in the paper. 


\section{REFERENCES}

1. G. Das and P. C. Mohapatra, Necessary and sufficient conditions for absolute Nörlund summability of Fourier series, Proc. London Math. Soc. (3) 41 (1980), 217-253.

2. H. P. Dikshit and A. Kumar, Absolute total-effectiveness of $\left(N, p_{n}\right)$ means I, Math. Proc. Cambridge Philos. Soc. 77 (1975), 103-108.

3. M. Izumi and S. Izumi, Absolute Nörlund summability of Fourier series of functions of bounded variation, Bull. Austral. Math. Soc. 3 (1970), 111-123.

4. W. B. Jurkat, A.Kumar, and A. Peyerimhoff, On the absolute Fourier-effectiveness of summability methods, J. London Math. Soc. (2) 31 (1985), 101-114.

5. Arun Kumar, Necessary and sufficient type theorem for absolute Nörlund summability of conjugate series, Proc. Amer. Math. Soc. 67 (1977), 77-83.

6. B. Kuttner and B. N. Sahney, On the absolute matrix summability of Fourier series, Pacific J. Math. 43 (1972), 407-419.

7. T. Pati, On the absolute Nörlund summability of the conjugate series of a Fourier series, J. London Math. Soc. (2) 38 (1963), 204-214.

Department of Mathematics, Rani Durgavati University, Jabalpur, India

Department of Mathematics, Ulm University, Ulm, Germany 